\title{
Variation in the peroxisome proliferator-activated receptor $\delta$ gene in relation to common metabolic traits in 7,495 middle-aged white people
}

\author{
N. Grarup • A. Albrechtsen • J. Ek • K. Borch-Johnsen • \\ T. Jørgensen • O. Schmitz • T. Hansen • O. Pedersen
}

Received: 22 December 2006 / Accepted: 28 February 2007 /Published online: 13 April 2007

(C) Springer-Verlag 2007

\begin{abstract}
Aims/hypothesis Studies in animals reveal that peroxisome proliferator-activated receptor $\delta(\operatorname{PPAR} \delta)$ regulates glucose metabolism and insulin sensitivity in both the liver and skeletal muscles. Moreover, $\operatorname{PPAR} \delta$ augments physical
\end{abstract}

Electronic supplementary material The online version of this article (doi:10.1007/s00125-007-0668-2) contains supplementary material, which is available to authorised users.

N. Grarup $(\varangle) \cdot$ A. Albrechtsen · J. Ek · K. Borch-Johnsen •

T. Hansen $\cdot O$. Pedersen

Steno Diabetes Center,

Niels Steensens Vej 1, NLC2.14,

2820 Gentofte, Copenhagen, Denmark

e-mail: ngrp@steno.dk

N. Grarup $\cdot$ K. Borch-Johnsen • O. Pedersen

Faculty of Health Sciences, University of Aarhus,

Aarhus, Denmark

\author{
A. Albrechtsen \\ Department of Biostatistics, University of Copenhagen, \\ Copenhagen, Denmark \\ J. Ek \\ Kennedy Institute-National Eye Clinic, \\ Glostrup, Denmark \\ K. Borch-Johnsen • T. Jørgensen \\ Research Centre for Prevention and Health, \\ Glostrup University Hospital, \\ Glostrup, Denmark \\ O. Schmitz \\ Department of Endocrinology and Diabetes M, \\ Aarhus University Hospital, \\ Aarhus, Denmark

\section{O. Schmitz} \\ Department of Clinical Pharmacology, University of Aarhus, \\ Aarhus, Denmark
}

endurance and increases oxidative metabolism, thereby averting obesity. Thus, we hypothesised that common variation in the PPARD gene is associated with insulin resistance and metabolic traits.

Materials and methods We studied variation in the exonic region of PPARD. Based upon the results of variant detection and information derived from the HapMap data resource, we selected common variants and tag singlenucleotide polymorphisms for genotyping in 7,495 white subjects, including 1,416 patients with type 2 diabetes.

Results Fourteen nucleotide variants were identified and a total of 12 variants capturing the common variation of PPARD were genotyped. In the population-based Inter99 (ClinicalTrials.gov ID no: NCT00289237) sample we observed no robust association with homeostasis model assessment of insulin resistance (HOMA-IR), adiposity measures or fasting serum lipids. Similarly, no association with type 2 diabetes or the metabolic syndrome was found. Conclusions/interpretation Based on thorough investigation, we conclude that common variation in PPARD does not significantly affect the risk of metabolic disease in the population studied. Given the confidence intervals that were found for effect size estimates, we can effectively rule out an increase in HOMA-IR of any tag SNP above 7\% per allele, assuming an additive model. Likewise, we can exclude an odds ratio of type 2 diabetes above 1.27 per allele.

Keywords Genetic epidemiology - Insulin resistance · Glucose · PPAR delta P PARD - Type 2 diabetes .

Metabolic syndrome

\footnotetext{
Abbreviations

HOMA-IR homeostasis model assessment of insulin resistance
} 
LD linkage disequilibrium

MAF minor allele frequency

PPAR peroxisome proliferator-activated receptor

SNP single-nucleotide polymorphism

\section{Introduction}

The pathogenesis of type 2 diabetes involves complex interaction of both genetic and behavioural factors. Besides glucose intolerance, many patients with type 2 diabetes have several additional independent risk factors for cardiovascular disease, including insulin resistance, dyslipidaemia, obesity and hypertension, together known as the metabolic syndrome [1].

The peroxisome proliferator-activated receptors (PPARs) belong to a nuclear receptor superfamily of ligand-inducible transcription factors. Three subtypes, $\alpha, \gamma$ and $\delta$, have been identified, with distinct roles in metabolism. PPAR $\alpha$ is a regulator of fatty acid oxidation, primarily produced in the liver [2], whereas PPAR $\gamma$ mainly functions as a regulator of adipogenesis [3]. In recent years, PPAR $\delta$ has emerged as a key player in the regulation of energy metabolism and as a potential novel drug target. PPAR $\delta$ is widely produced and VLDL-derived fatty acids serve as endogenous receptor agonists enhancing the expression of target genes $[4,5]$. $\operatorname{PPAR} \delta$ acts as a regulator of fatty acid $\beta$-oxidation in both adipose tissue and skeletal muscle. In skeletal muscle, overexpression of Ppard in mice increases oxidative metabolism, changes muscle fibre type towards oxidative type I fibres and improves physical endurance thereby averting obesity induced by a high-fat diet or genetically $[6$, 7]. Additionally, a recent study of genetically modified mice provides evidence that $\operatorname{PPAR} \delta$ regulates glucose metabolism and insulin sensitivity in both skeletal muscle and the liver, interestingly indicating a subtle $\operatorname{PPAR} \delta$ controlled change in substrate utilisation [8]. Increased Ppard expression in adipose tissue in mice is associated with reduced adiposity and lower serum lipid levels $[9,10]$. In addition, activation of $\operatorname{PPAR} \delta$ by treatment with a synthetic agonist ameliorates diet-induced obesity and insulin resistance in mice [10] and improves circulating lipid profiles in both obese rhesus monkeys and $d b / d b$ mice $[11,12]$. In line with the outcome of studies in rodents, expression profiling studies in humans have shown an increase in PPARD expression following endurance exercise $[13,14]$. Together, these studies point to a critical role for PPAR $\delta$ in the regulation of metabolic traits and cardiovascular risk factors.

PPARD is located on chromosome $6 \mathrm{p} 21$, a $85 \mathrm{~kb}$ region which has not shown linkage to insulin resistance, obesity or type 2 diabetes. Previous studies of the variation in
PPARD in relation to features of the metabolic syndrome have resulted in conflicting results. Several studies including fewer than 1,000 participants have investigated the $\mathrm{c}$. $-87 \mathrm{~T} \rightarrow \mathrm{C}$ (rs2016520) variant in exon 4 in relation to serum lipid profiles and have shown a positive association of the minor $\mathrm{C}$ allele with higher serum levels of LDL-cholesterol [15], lower HDL-cholesterol [16, 17], higher triacylglycerol [18] or no association [19-21]. In addition, a recent paper reported that three variants in PPARD and a haplogenotype of these variants were associated with increased insulinstimulated whole-body and skeletal muscle glucose uptake in a study including 129 carefully phenotyped subjects [22]. Similarly, a Finnish study of 769 middle-aged people demonstrated an increased risk of conversion from IGT to overt type 2 diabetes during a 5-year follow-up period in carriers of the rs6902123 variant [23].

Since PPARD represents an obvious biological candidate gene in the regulation of metabolic traits, we have systematically investigated variation in PPARD and genotyped tag single-nucleotide polymorphisms (SNPs) in a relatively large sample of white middle-aged subjects. We primarily hypothesised that variation in PPARD influences levels of insulin resistance, fasting serum lipid profiles, fasting glycaemia and adiposity. In addition, we related genotypes to type 2 diabetes and the metabolic syndrome.

\section{Subjects and methods}

Subjects Mutation analysis was carried out in 60 patients with type 2 diabetes recruited at the Steno Diabetes Center $\left(\right.$ mean \pm SD age $67.1 \pm 10$ years; BMI $26.9 \pm 6.9 \mathrm{~kg} / \mathrm{m}^{2}$; $\mathrm{HbA}_{1 \mathrm{c}} 8.1 \pm 1.8 \%$ ), ten healthy subjects selected for low physical fitness (age 28.1 \pm 3.2 years; BMI $31.7 \pm 5.9 \mathrm{~kg} / \mathrm{m}^{2}$; $V \mathrm{O}_{2 \max } 23.2 \pm 2.0 \mathrm{ml} \mathrm{O}_{2} \mathrm{~kg}^{-1} \mathrm{~min}^{-1}$ ) and 11 healthy subjects selected for high physical fitness (age 24.0 \pm 3.7 years; BMI $\left.21.5 \pm 2.6 \mathrm{~kg} / \mathrm{m}^{2} ; V \mathrm{O}_{2 \max } 63.4 \pm 4.3 \mathrm{ml} \mathrm{O} \mathrm{kg}^{-1} \mathrm{~min}^{-1}\right)$.

Twelve PPARD polymorphisms were genotyped in 7,495 white subjects in three study groups. Group 1 was a population-based, randomised, non-pharmacological intervention study for the prevention of ischemic heart disease (Inter99; ClinicalTrials.gov ID no: NCT00289237) conducted at the Research Centre for Prevention and Health in Glostrup, Copenhagen (3,028 men, 3,043 women; mean \pm SD age $46.2 \pm 7.9$ years; BMI $26.3 \pm 4.6 \mathrm{~kg} / \mathrm{m}^{2}$ ) [24]. Of the 6,071 participants with available genomic DNA, 4,543 (75\%) had NGT, 503 (8.3\%) had IFG, 693 (11\%) had IGT, $252(4.2 \%)$ had screen-detected and treatment-naive type 2 diabetes, and $100(1.6 \%)$ had previously diagnosed type 2 diabetes. Group 2 consisted of 360 unrelated and elderly subjects (174 men, 186 women; mean $\pm \mathrm{SD}$ age $62.0 \pm$ 4.5 years; BMI $26.1 \pm 3.7 \mathrm{~kg} / \mathrm{m}^{2}$ ) with NGT recruited at random from Copenhagen County. Group 3 consisted of 
1,064 unrelated patients with type 2 diabetes (642 men, 422 women; mean \pm SD age $58.9 \pm 10.7$ years; BMI $29.5 \pm 5.2 \mathrm{~kg} /$ $\mathrm{m}^{2}$ ) recruited at the outpatient clinic at Steno Diabetes Center, Copenhagen. All subjects of study groups 1 and 2 underwent a standard $75 \mathrm{~g}$ OGTT.

The study of quantitative metabolic traits was performed in the Inter99 cohort (study group 1), excluding patients treated for type 2 diabetes, and included a total of 5,971 subjects. Subgroup analyses involved 1,448 participants with treatment-naive impaired glucose regulation, i.e. IFG, IGT or screen-detected type 2 diabetes from the Inter99 cohort (870 men, 578 women; mean \pm SD age $49.0 \pm$ 7.3 years; BMI $28.4 \pm 5.1 \mathrm{~kg} / \mathrm{m}^{2}$ ). Subjects treated with lipid-lowering drugs $(n=89)$ were excluded in all analyses of fasting serum lipids.

All patients with type 2 diabetes and subjects with NGT were included in the association study of type 2 diabetes, which involved a total of $1,416(60 \%$ men) unrelated patients with type 2 diabetes and 4,883 (47\% men) unrelated control subjects with NGT. The phenotypic characteristics of the patients were as follows: mean $\pm \mathrm{SD}$ age at entry to the examination $56.9 \pm 10.5$ years; age at clinical diagnosis $51.9 \pm 10.6$ years; BMI $29.7 \pm 5.3 \mathrm{~kg} / \mathrm{m}^{2}$; and $\mathrm{HbA}_{1 \mathrm{c}} 7.8 \pm 1.7 \%$. Diabetes and the prediabetic states were diagnosed according to the WHO 1999 criteria [25]. At recruitment, diabetes patients with one or more of the following characteristics were excluded: (1) diabetes due to known chronic pancreatitis, haemochromatosis, severe insulin resistance, MODY, or maternally inherited diabetes and deafness; (2) a family history of first-degree relatives with type 1 diabetes; (3) a requirement for insulin within the first year after the diagnosis of diabetes; or (4) a fasting serum C-peptide level $\leq 150 \mathrm{pmol} / \mathrm{l}$. The phenotypic characteristics of the control subjects, who had both normal fasting glycaemia and normal glucose tolerance, were as follows: age $46.4 \pm 8.8$ years (mean \pm SD); BMI 25.6 \pm $4.1 \mathrm{~kg} / \mathrm{m}^{2}$.

All participants were evaluated in a case-control study examining the association between genotype and the metabolic syndrome according to the WHO 1999 definition [25]. Subjects having no components of the metabolic syndrome were considered control subjects.

Exact numbers of subjects included in the individual studies are given in the tables.

All study participants were white by self-report. Informed written consent was obtained from all study participants. The studies were conducted in accordance with the Declaration of Helsinki II and were approved by the local Ethical Committee of Copenhagen.

Biochemical and anthropometric measures Height and weight were measured in light indoor clothing and without shoes, and BMI was calculated as weight $(\mathrm{kg}) /$ height $^{2}\left(\mathrm{~m}^{2}\right)$ [24]. Waist circumference was measured in the upright position midway between the iliac crest and the lower costal margin, and hip circumference was measured at its maximum. Blood samples were drawn after a $12-\mathrm{h}$ overnight fast. Plasma glucose was analysed by a glucose oxidase method (Granutest; Merck, Darmstadt, Germany) and serum insulin (excluding des-31,32 and intact proinsulin) was measured using the Autodelfia insulin kit (PerkinElmer/Wallac, Turku, Finland). Serum triacylglycerol and serum total and HDL-cholesterol were analysed using enzymatic colorimetric methods (GPO-PAP and CHODPAP; Roche Molecular Biochemicals, Mannheim, Germany). LDL-cholesterol was estimated using Friedewald's equation [26]. $\mathrm{HbA}_{1 \mathrm{c}}$ was measured by ion-exchange HPLC (normal range 4.1-6.4\%). Homeostasis model assessment of insulin resistance (HOMA-IR) was calculated as $\mathrm{HOMA}-\mathrm{IR}=($ fasting plasma - glucose $[\mathrm{mmol} / \mathrm{l}] \times$ fasting serum - insulin $[\mathrm{pmol} / 1]) / 22.5$ [27].

Mutation analysis The mutation analysis for novel variants in the PPARD promoter and all nine exons including intron-exon boundaries was carried out using denaturing HPLC and subsequent direct nucleotide sequencing (carried out by MWG Biotech, Ebersberg, Germany) on genomic DNA purified from human leucocytes. The denaturing HPLC method has a sensitivity $>95 \%$ [28]. We had $99.9 \%$ statistical power to detect variants with a minor allele frequency (MAF) above 5\%. Primer sequences are available upon request.

Tag SNP selection We genotyped common variants (MAF $>5 \%$ ) identified in the mutation analysis and selected additional tag SNPs obtained from the HapMap data resource of Utah residents with ancestry from northern and western Europe (HapMap CEU population) (www. hapmap.org, release 19/phase II) [29]. Tag SNPs were selected using the Carlson greedy algorithm [30] capturing all SNPs with MAF $\geq 5 \%$ at a minimum $R^{2}$ of 0.8 . We did not attempt to cover rare variation (MAF $<5 \%$ ) since our sample size did not have sufficient statistical power to detect modest effect sizes of rare alleles.

Genotyping Genotyping of 12 SNPs in PPARD (rs34474204, rs7758272, rs6902123, rs9470001, rs6457816, rs9658119, rs9380506, rs9470015, rs2016520, rs4713854, rs2076169 and rs2076167) was performed by TaqMan allelic discrimination (KBiosciences, Hoddesdon, UK). All genotyping success rates were above $94 \%$. For each variant, 469 samples were genotyped in duplicate, with a mismatch rate below $0.8 \%$. The distributions of genotypes for all variants were in Hardy-Weinberg equilibrium (Electronic supplementary material [ESM] Table 1). 
Statistical analysis A general linear model was used for testing quantitative traits in relation to genotype, adjusting for the effects of sex, age and BMI, when appropriate. Quantitative traits were checked for normality of the residuals and, if appropriate, logarithmically transformed. Linkage disequilibrium (LD) between markers and haplotype blocks was evaluated using Haploview version 4.0, beta10 (http://www.broad.mit.edu/mpg/haploview/). Haplotype frequencies were estimated using the EM algorithm and association with quantitative traits was evaluated by global and haplotype-specific score statistics and simulation [31], while the effect sizes of each haplotype were estimated by modelling the haplotype-phenotype interaction [32]. In the association studies of type 2 diabetes and the metabolic syndrome, Fisher's exact test was used to examine differences in allele frequencies between cases and control subjects and logistic regression was used to examine differences in genotypes assuming an additive, a dominant or a recessive model with adjustment for sex, age and BMI. A $p$ value of less than 0.05 was considered significant. Permutation testing of individual quantitative traits to obtain an empirical $p$ value was performed using Blossom version 2005.11.23 (available at http://www.fort.usgs.gov/Products/ Software/Blossom/). For all traits tested, 100,000 permutations were done [33]. All other analyses were performed using RGui version 2.2.4 (available at http://www.r-project. org). Bonferroni correction for multiple hypothesis testing was applied using Nyholt's method [34] to estimate the effective number of independent markers.

\section{Results}

We performed mutation analysis of all nine exons, intronexon boundaries and $800 \mathrm{bp}$ of the putative promoter and identified 14 variants in PPARD; four had a heterozygosity rate above 10\% (ESM Table 2). Only two intronic variants were not previously reported and both were found in only a single carrier and were not investigated further. We selected common variants from the mutation analysis and additional tag SNPs from the HapMap CEU panel and genotyped 12 markers (ESM Table 1).

Initially we analysed quantitative metabolic traits reflecting levels of insulin sensitivity, fasting serum lipids, fasting glycaemia and adiposity in 5,971 subjects with NGT, IFG, IGT or screen-detected, treatment-naive type 2 diabetes from the population-based Inter99 cohort. We found a borderline statistically significant association of the rare allele of rs9658119 with decreased insulin sensitivity assessed by HOMA-IR $(p=0.05)$ (Table 1$)$. Furthermore, the rare alleles of the rs9470001, rs6457816 and rs9658119 variants associated nominally with increased fasting plasma glucose ( $p<0.05$, data not shown). We showed no association of tag SNPs with fasting serum lipids (data not shown) or adiposity measures (Table 2). All analyses were confirmed by permutation test of individual traits to obtain an empirical $p$ value and consistency was observed. However, when correcting for multiple hypothesis testing using Bonferroni correction taking LD between markers into account [34], none of these associations were statistically significant at the study-wide level.

Table 1 HOMA-IR (pmol/l×mmol/l) in middle-aged white subjects with NGT, IFG, IGT or screen-detected and treatment-naive type 2 diabetes recruited from the Inter99 cohort and stratified according to genotype of PPARD tag SNPs

\begin{tabular}{|c|c|c|c|c|c|}
\hline \multirow[t]{2}{*}{ SNP } & \multirow[t]{2}{*}{$n(\mathrm{Wt}, \mathrm{He}, \mathrm{Ho})$} & \multicolumn{4}{|c|}{ HOMA-IR (pmol/1×mmol/l) } \\
\hline & & $\mathrm{Wt}$ & $\mathrm{He}$ & Ho & $p$ value \\
\hline rs 34474204 & $5,731(5,135,585,11)$ & $10.5 \pm 7.9$ & $11.2 \pm 8.7$ & $7.86 \pm 5.3$ & 0.79 \\
\hline rs7758272 & $5,607(5,011,580,16)$ & $10.5 \pm 7.9$ & $11.2 \pm 8.7$ & $7.48 \pm 4.5$ & 0.61 \\
\hline rs6902123 & $5,742(5,006,710,26)$ & $10.5 \pm 7.9$ & $11.3 \pm 8.8$ & $8.72 \pm 5.7$ & 0.39 \\
\hline rs9470001 & $5,723(4,990,708,25)$ & $10.5 \pm 7.9$ & $11.4 \pm 8.9$ & $9.28 \pm 6.5$ & 0.34 \\
\hline rs6457816 & $5,729(4,976,726,27)$ & $10.5 \pm 7.9$ & $11.3 \pm 8.7$ & $9.39 \pm 5.6$ & 0.38 \\
\hline rs9658119 & $5,746(4,689,999,58)$ & $10.4 \pm 7.8$ & $11.0 \pm 8.2$ & $12.2 \pm 10.7$ & 0.05 \\
\hline rs9380506 & $5,764(4,640,1,077,47)$ & $10.6 \pm 7.9$ & $10.5 \pm 8.3$ & $10.1 \pm 6.2$ & 0.20 \\
\hline rs9470015 & $5,754(4,190,1,448,116)$ & $10.6 \pm 8.0$ & $10.5 \pm 7.7$ & $11.3 \pm 8.5$ & 0.82 \\
\hline rs 2016520 & $5,712(4,012,1,575,125)$ & $10.6 \pm 8.2$ & $10.5 \pm 7.7$ & $10.7 \pm 6.8$ & 0.9 \\
\hline rs4713854 & $5,756(4,860,855,41)$ & $10.5 \pm 8.0$ & $10.7 \pm 7.9$ & $11.3 \pm 7.2$ & 0.37 \\
\hline rs2076169 & $5,732(4,737,949,46)$ & $10.6 \pm 8.0$ & $10.6 \pm 7.6$ & $12.0 \pm 7.5$ & 0.44 \\
\hline rs2076167 & $5,739(3,721,1,799,219)$ & $10.5 \pm 7.9$ & $10.7 \pm 8.0$ & $11.2 \pm 7.4$ & 0.41 \\
\hline
\end{tabular}

Data are mean $\pm \mathrm{SD}$

$n$ number of subjects successfully genotyped for the given variant, with numbers of subjects wild-type (Wt), heterozygous $(H e)$ or homozygous $\mathrm{Ho}$ ) for the variant in parentheses

The $p$ values were calculated using a general linear model assuming an additive model and were adjusted for the effects of age, sex and BMI The dependent variable was logarithmically transformed before analysis 
Table 2 BMI in middle-aged white subjects with NGT, IFG, IGT or screen-detected and treatment-naive type 2 diabetes recruited from the Inter99 cohort and stratified according to genotype of PPARD tag SNPs

\begin{tabular}{|c|c|c|c|c|c|}
\hline \multirow[t]{2}{*}{ SNP } & \multirow[t]{2}{*}{$n(\mathrm{Wt}, \mathrm{He}, \mathrm{Ho})$} & \multicolumn{4}{|c|}{ BMI $\left(\mathrm{kg} / \mathrm{m}^{2}\right)$} \\
\hline & & $\mathrm{Wt}$ & $\mathrm{He}$ & Ho & $p$ value \\
\hline rs34474204 & $5,732(5,136,585,11)$ & $26.2 \pm 4.5$ & $26.4 \pm 4.8$ & $24.4 \pm 4.1$ & 0.47 \\
\hline rs7758272 & $5,608(5,012,580,16)$ & $26.2 \pm 4.5$ & $26.6 \pm 5.0$ & $23.9 \pm 4.0$ & 0.16 \\
\hline rs6902123 & $5,743(5,007,710,26)$ & $26.2 \pm 4.5$ & $26.4 \pm 4.8$ & $25.4 \pm 4.4$ & 0.35 \\
\hline rs9470001 & $5,724(4,991,708,25)$ & $26.2 \pm 4.5$ & $26.5 \pm 4.8$ & $25.5 \pm 5.1$ & 0.21 \\
\hline rs6457816 & $5,730(4,977,726,27)$ & $26.2 \pm 4.5$ & $26.5 \pm 4.9$ & $25.5 \pm 4.5$ & 0.25 \\
\hline rs9658119 & $5,747(4,689,1,000,58)$ & $26.2 \pm 4.5$ & $26.3 \pm 4.8$ & $27.0 \pm 4.9$ & 0.38 \\
\hline rs9380506 & $5,765(4,640,1,078,47)$ & $26.2 \pm 4.5$ & $26.2 \pm 4.6$ & $26.4 \pm 4.7$ & 0.72 \\
\hline rs9470015 & $5,755(4,190,1,449,116)$ & $26.2 \pm 4.5$ & $26.2 \pm 4.5$ & $26.7 \pm 5.1$ & 0.31 \\
\hline rs2016520 & $5,713(4,012,1,576,125)$ & $26.2 \pm 4.5$ & $26.2 \pm 4.5$ & $26.5 \pm 5.0$ & 0.55 \\
\hline rs4713854 & $5,757(4,860,856,41)$ & $26.2 \pm 4.5$ & $26.3 \pm 4.6$ & $27.0 \pm 4.9$ & 0.24 \\
\hline rs2076169 & $5,733(4,737,950,46)$ & $26.2 \pm 4.5$ & $26.2 \pm 4.5$ & $26.6 \pm 5.1$ & 0.89 \\
\hline rs2076167 & $5,740(3,721,1,800,219)$ & $26.2 \pm 4.5$ & $26.3 \pm 4.6$ & $26.5 \pm 4.9$ & 0.29 \\
\hline
\end{tabular}

Data are mean $\pm \mathrm{SD}$

$n$ designates the number of subjects successfully genotyped for the given variant with numbers of subjects wild-type $(\mathrm{Wt})$, heterozygous $(\mathrm{He})$ or homozygous $(\mathrm{Ho})$ for the variant in parentheses

The $p$ values were calculated using a general linear model assuming an additive model and were adjusted for the effect of age and sex

We investigated LD between genotyped variants and constructed haplotype blocks based on confidence bounds of LD measured by $\mathrm{D}^{\prime}$ [35]. Three blocks were detected (Fig. 1) and we tested haplotypes of these three blocks for association with measures of insulin resistance, fasting serum lipids, fasting glycaemia and adiposity in 5,971 subjects. No haplotype in block 1 or 2 associated with quantitative metabolic traits (data not shown); however, in block 3 a haplotype consisting of the rare alleles of both variants was nominally associated with increased incremental area under the serum insulin curve during an OGTT (global $p=0.02$, haplotype-specific $p=0.02$ ). Interestingly,

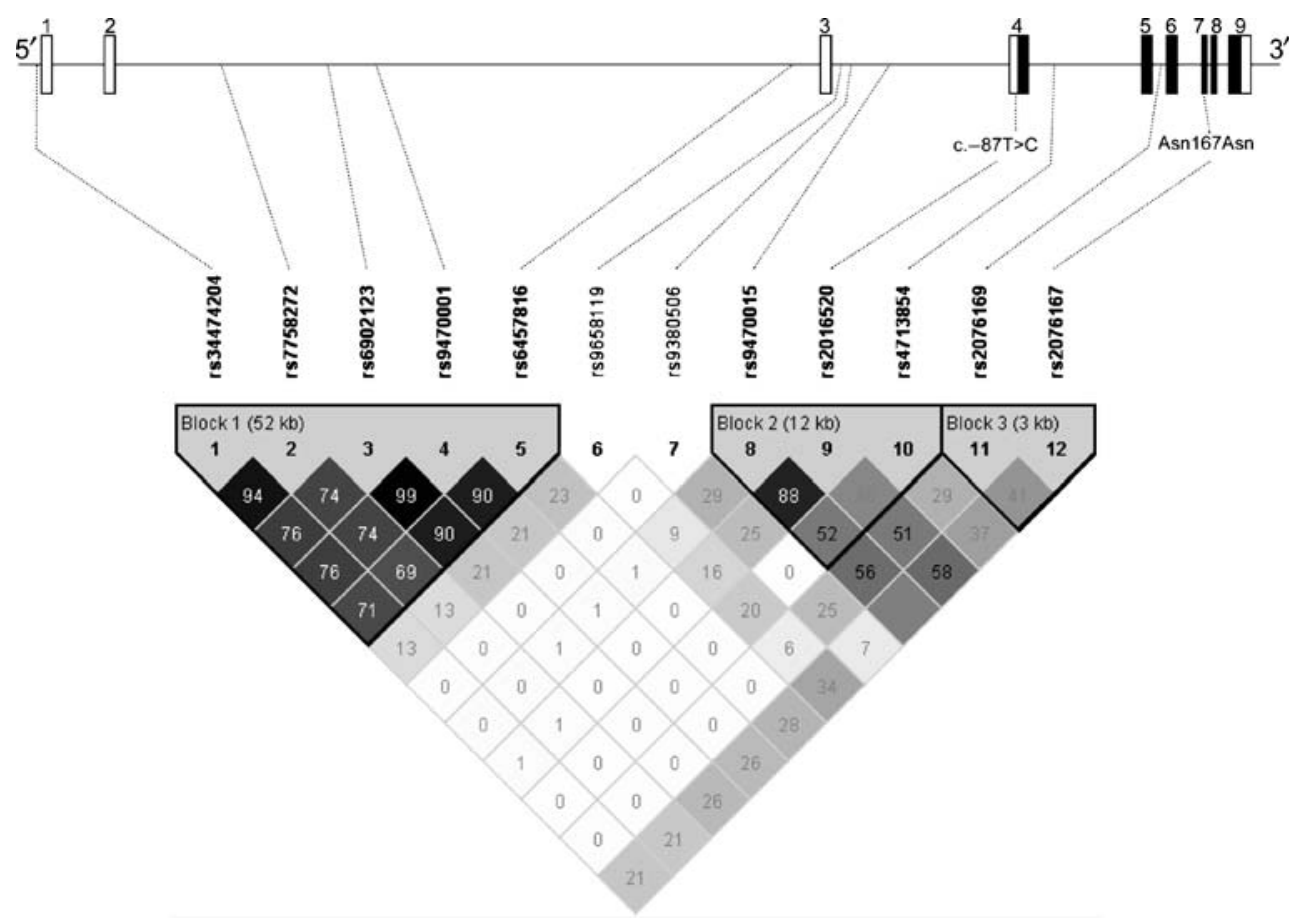

Fig. 1 Gene structure of PPARD, localisation of the genotyped tag SNPs and pairwise LD estimated by $R^{2}$ between tag SNPs. Exons are numbered. Coding sequence is shown in black. Numbers in squares designate the degree of $\mathrm{LD}\left(R^{2}\right)$ between any two markers. Haplotype blocks were constructed based on confidence bounds of LD measured by $\mathrm{D}^{\prime}$ [35]. LD estimates and haplotype blocks were made using Haploview version 4.0, beta10 (http://www.broad.mit.edu/mpg/haploview/) 
analysing a subgroup consisting of 1,448 treatment-naive subjects with impaired glucose regulation (IFG, IGT or screen-detected type 2 diabetes) strengthened the association of this haplotype with traits reflecting insulin sensitivity, as we detected an association with increased HOMA-IR (global $p=0.001$; Table 3). To ensure the robustness of the $p$ values, haplotype associations were tested using simulation and all results were consistent. However, after correction for multiple hypothesis testing none of the described associations were statistically significant at the study-wide level (haplotype association with HOMA-IR [Table 3], $p=0.14$ ).

Examining 1,416 patients with type 2 diabetes and 4,883 control subjects, we were unable to demonstrate any association of PPARD single variants or haplotypes with type 2 diabetes (ESM Table 3). Additionally, we found no differences in allele frequencies of variants in PPARD between 2,315 cases with WHO-defined metabolic syndrome [25] and 1,925 control subjects without any component of the metabolic syndrome (data not shown).

\section{Discussion}

In recent years $\operatorname{PPAR} \delta$ has emerged as a key protein in the regulation of energy metabolism by its ability to enhance fatty acid catabolism, energy uncoupling and insulin sensitivity in the liver, adipose tissue and skeletal muscle $[6-10,36]$.

In the present study we systematically investigated variation in PPARD by mutation analysis and subsequent selection of additional tag SNPs based on the HapMap phase II CEU panel. Twelve variants were genotyped in large samples. We found no solid association with the insulin resistance index obtained from OGTT data when we analysed single variants and haplotypes in subjects with NGT, IFG, IGT or screen-detected, treatment-naive type 2 diabetes. Based on $95 \%$ confidence intervals of estimates of HOMA-IR, we can exclude an increase of more than $7 \%$ per allele assuming an additive model. However, subgroup analysis of 1,448 middle-aged white subjects with treatment-naive impaired glucose regulation (IFG, IGT or screen-detected and treatment-naive type 2 diabetes) demonstrated a significant association of a haplotype in block 3 with decreased insulin sensitivity as assessed by HOMAIR. However, it is recognised that since analysis in this particular study subgroup was not a part of the a priori defined set of hypotheses and because the association was not statistically significant after stringent correction for the testing of multiple hypotheses, these findings are merely exploratory and need replication.

A previous study demonstrated a statistically significant increase in insulin-stimulated whole-body and skeletal muscle glucose uptake assessed by the hyperinsulinaemiceuglycaemic clamp technique in carriers of the rare alleles of three variants in PPARD and carriers of a genotype combination of these variants, in studies of 129 young, healthy and glucose-tolerant Finnish subjects [22]. The association was strongest for the rs6902123 variant; however, in the present study we failed to replicate this finding. The reason for this disparity is not clear. However, it is emphasised that application of a more relevant and precise measure of insulin sensitivity in the present protocol, i.e. the hyperinsulinaemic-euglycaemic clamp technique, may have changed the results. Besides, we appreciate that both genetic and environmental heterogeneity, including differences in their interaction, could give rise to population-specific discrepancies in the association of allelic variants and insulin resistance and thereby account for the inconsistent findings.

Previous studies of variants in PPARD have provided conflicting results regarding association with metabolic traits. The minor allele of the rs2016520 variant in exon 4 has inconsistently been associated with decreased fasting levels of HDL-cholesterol [15], increased LDL-cholesterol

Table 3 Haplotypes in haplotype block 3 in relation to homeostasis model assessment of insulin resistance in 1,448 middle-aged white

impaired glucose regulation (IFG, IGT or screen-detected and treatment-naive type 2 diabetes) subjects recruited from the Inter99 cohort with treatment-naive

\begin{tabular}{|c|c|c|c|c|c|c|}
\hline Haplotype & rs2076169 & rs2076167 & Frequency & Estimate $\pm \mathrm{SE}^{\mathrm{a}}$ & $p$ value & $p_{\mathrm{SIM}}$ \\
\hline Global score statistics & & & & & 0.001 & 0.001 \\
\hline 1 & $\mathrm{~A}$ & $\mathrm{C}$ & $80 \%$ & & 0.006 & 0.008 \\
\hline 2 & $\mathrm{~A}$ & $\mathrm{~T}$ & $11 \%$ & $0.067 \pm 0.033$ & 0.07 & 0.07 \\
\hline 3 & $\mathrm{G}$ & $\mathrm{T}$ & $9 \%$ & $0.080 \pm 0.036$ & 0.04 & 0.04 \\
\hline
\end{tabular}

${ }^{\text {a }}$ Regression estimate \pm SE of the logarithm of HOMA-IR (pmol/1 $\left.\times \mathrm{mmol} / \mathrm{l}\right)$ with haplotype 1 (A-C) as base

Haplotype frequencies were estimated using the EM algorithm and association with quantitative traits was evaluated by global and haplotypespecific score statistics ( $p$ value) and simulation $\left(p_{\text {SIM }}\right)$ [31]

Estimates of the effect sizes of each haplotype were estimated by modelling the haplotype xphenotype interaction [32] 
$[16,17]$ and increased fasting plasma glucose [37] or decreased BMI [20], even though some studies found no association $[19,21]$. Yet all studies were probably statistically underpowered, involving fewer than 1,000 participants. Despite the relatively large sample size in the present study, we did not replicate any of these associations of rs2016520 with metabolic traits and we detected no association of PPARD tag SNPs or haplotypes with fasting serum lipids or measures of adiposity. We did, however, find nominally statistical significant associations with increased fasting plasma glucose. The present study is, so far, the largest study to investigate variation in PPARD in relation to quantitative metabolic traits and the design has the advantage of allowing estimation of the genotypic effect at the population level of middle-aged people.

A recent study demonstrated an increased risk of conversion to overt type 2 diabetes in carriers of the rs6902123 variant when 679 subjects with IGT were analysed [23]; however, a cross-sectional study observed no association of rs2016520 genotype with type 2 diabetes in 402 patients with type 2 diabetes and 436 control subjects [19]. Based on confidence intervals of estimates of effect sizes of type 2 diabetes, we can exclude an odds ratio above 1.27 per allele assuming an additive model.

In the present study we aimed to cover variation in PPARD on a gene-wide basis and therefore engaged in both de novo mutation analysis and selected additional tag SNPs. Although most studies show reasonable transferability of tag SNPs selected from the HapMap data resource to other populations $[38,39]$, it is possible that allele frequencies of and LD structure between variants are different in the study sample, leading to impaired performance of the tag SNPs. In addition, we do appreciate that we did not attempt to capture rare variation (MAF below $5 \%$ ) in PPARD, which may influence metabolic phenotypes significantly.

In conclusion, in the present study of tag SNPs in PPARD in relation to common metabolic phenotypes we did not demonstrate any consistent associations of genotypes or haplotypes with measures of insulin sensitivity, fasting serum lipid levels, adiposity or type 2 diabetes. Yet subgroup analysis showed a putative impact of a PPARD haplotype on the HOMA insulin sensitivity index in treatment-naive subjects with impaired glucose regulation. However, the association was not significant after stringent correction for multiple hypothesis testing.

Acknowledgements The authors wish to thank A. Forman, I.-L. Wantzin and M. Stendal for technical assistance and G. Lademann for secretarial support. The study was supported by grants from the European Union (EXGENESIS, grant no. LSHM-CT-2004-005272), the FOOD Study Group/the Danish Ministry of Food, Agriculture and Fisheries \& Ministry of Family and Consumer Affairs (grant no. 210105-0044), the Faculty of Health Sciences of Aarhus University, the
Danish Clinical Intervention Research Academy, the Danish Diabetes Association and the Sehested Hansen Foundation.

Duality of interest K. Borch-Johnsen holds stock in Novo Nordisk and has received lecture fees from pharmaceutical companies. All other authors declare no duality of interest.

\section{References}

1. Andersen G, Hansen T, Pedersen O (2005) Genetics of common forms of glycaemia with pathological impact on vascular biology: are we on the right track? Curr Mol Med 5:261-274

2. Lefebvre P, Chinetti G, Fruchart JC, Staels B (2006) Sorting out the roles of PPAR $\alpha$ in energy metabolism and vascular homeostasis. J Clin Invest 116:571-580

3. Semple RK, Chatterjee VK, O'Rahilly S (2006) PPAR $\gamma$ and human metabolic disease. J Clin Invest 116:581-589

4. Chawla A, Lee CH, Barak Y et al (2003) PPAR $\alpha$ is a very lowdensity lipoprotein sensor in macrophages. Proc Natl Acad Sci USA 100:1268-1273

5. Lee CH, Kang K, Mehl IR et al (2006) Peroxisome proliferatoractivated receptor $\delta$ promotes very low-density lipoproteinderived fatty acid catabolism in the macrophage. Proc Natl Acad Sci USA 103:2434-2439

6. Wang YX, Zhang CL, Yu RT et al (2004) Regulation of muscle fiber type and running endurance by PPAR $\delta$. PLoS Biol 2:e294

7. Luquet S, Lopez-Soriano J, Holst D et al (2003) Peroxisome proliferator-activated receptor delta controls muscle development and oxidative capability. FASEB J 17:2299-2301

8. Lee $\mathrm{CH}$, Olson P, Hevener A et al (2006) PPAR $\delta$ regulates glucose metabolism and insulin sensitivity. Proc Natl Acad Sci USA 103:3444-3449

9. Wang YX, Lee CH, Tiep S et al (2003) Peroxisome-proliferatoractivated receptor delta activates fat metabolism to prevent obesity. Cell 113:159-170

10. Tanaka T, Yamamoto J, Iwasaki S et al (2003) Activation of peroxisome proliferator-activated receptor delta induces fatty acid beta-oxidation in skeletal muscle and attenuates metabolic syndrome. Proc Natl Acad Sci USA 100:15924-15929

11. Oliver WR, Shenk JL, Snaith MR et al (2001) A selective peroxisome proliferator-activated receptor delta agonist promotes reverse cholesterol transport. Proc Natl Acad Sci USA 98:5306-5311

12. Leibowitz MD, Fiévet C, Hennuyer N et al (2000) Activation of PPAR $\delta$ alters lipid metabolism in $\mathrm{db} / \mathrm{db}$ mice. FEBS Lett 473:333-336

13. Watt MJ, Southgate RJ, Holmes AG, Febbraio MA (2004) Suppression of plasma free fatty acids upregulates peroxisome proliferator-activated receptor (PPAR) $\alpha$ and $\delta$ and PPAR coactivator $1 \alpha$ in human skeletal muscle, but not lipid regulatory genes. J Mol Endocrinol 33:533-544

14. Mahoney DJ, Parise G, Melov S, Safdar A, Tarnopolsky MA (2005) Analysis of global mRNA expression in human skeletal muscle during recovery from endurance exercise. FASEB J 19:1498-1500

15. Skogsberg J, McMahon AD, Karpe F, Hamsten A, Packard CJ, Ehrenborg E (2003) Peroxisome proliferator activated receptor delta genotype in relation to cardiovascular risk factors and risk of coronary heart disease in hypercholesterolaemic men. J Intern Med 254:597-604

16. Skogsberg J, Kannisto K, Cassel TN, Hamsten A, Eriksson P, Ehrenborg E (2003) Evidence that peroxisome proliferatoractivated receptor delta influences cholesterol metabolism in men. Arterioscler Thromb Vasc Biol 23:637-643 
17. Aberle J, Hopfer I, Beil FU, Seedorf U (2006) Association of the T+294C polymorphism in $\operatorname{PPAR} \delta$ with low HDL cholesterol and coronary heart disease risk in women. Int $\mathrm{J}$ Med Sci 3:108-111

18. Chen S, Tsybouleva N, Ballantyne CM, Gotto AM, Marian AJ (2004) Effects of PPARalpha, gamma and delta haplotypes on plasma levels of lipids, severity and progression of coronary atherosclerosis and response to statin therapy in the lipoprotein coronary atherosclerosis study. Pharmacogenetics 14:61-71

19. Gouni-Berthold I, Giannakidou E, Faust M, Berthold HK, Krone W (2005) The peroxisome proliferator-activated receptor delta $+294 \mathrm{~T} /$ $\mathrm{C}$ polymorphism in relation to lipoprotein metabolism in patients with diabetes mellitus type 2 and in non-diabetic controls. Atherosclerosis 183:336-341

20. Aberle J, Hopfer I, Beil FU, Seedorf U (2006) Association of peroxisome proliferator-activated receptor delta $+294 \mathrm{~T} / \mathrm{C}$ with body mass index and interaction with peroxisome proliferator-activated receptor alpha L162V. Int J Obes (Lond) 30:1709-1713

21. Robitaille J, Gaudet D, Perusse L, Vohl MC (2007) Features of the metabolic syndrome are modulated by an interaction between the peroxisome proliferator-activated receptor-delta $-87 \mathrm{~T}>\mathrm{C}$ polymorphism and dietary fat in French-Canadians. Int J Obes (Lond) 31:411-417

22. Vänttinen M, Nuutila $P$, Kuulasmaa $T$ et al (2005) Single nucleotide polymorphisms in the peroxisome proliferator-activated receptor $\delta$ gene are associated with skeletal muscle glucose uptake. Diabetes 54:3587-3591

23. Andrulionyte L, Peltola P, Chiasson JL, Laakso M (2006) Single nucleotide polymorphisms of $P P A R D$ in combination with the Gly482Ser substitution of $P G C-1 A$ and the Pro12Ala substitution of PPARG2 predict the conversion from impaired glucose tolerance to type 2 diabetes: the STOP-NIDDM trial. Diabetes 55:2148-2152

24. Jørgensen T, Borch-Johnsen K, Thomsen TF, Ibsen H, Glumer C, Pisinger C (2003) A randomized non-pharmacological intervention study for prevention of ischaemic heart disease: baseline results Inter99 (1). Eur J Cardiovasc Prev Rehab 10:377-386

25. World Health Organization Study Group (1999) Definition, diagnosis and classification of diabetes mellitus and its complications. Part 1: Diagnosis and classification of diabetes mellitus. Tech
Rep Ser WHO/NCD/NCS/99, 2nd edn. World Health Organization, Geneva

26. Friedewald WT, Levy RI, Fredrickson DS (1972) Estimation of the concentration of low-density lipoprotein cholesterol in plasma, without use of the preparative ultracentrifuge. Clin Chem 18:499-502

27. Matthews DR, Hosker JP, Rudenski AS, Naylor BA, Treacher DF, Turner RC (1985) Homeostasis model assessment: insulin resistance and beta-cell function from fasting plasma glucose and insulin concentrations in man. Diabetologia 28:412-419

28. Xiao W, Oefner PJ (2001) Denaturing high-performance liquid chromatography: a review. Hum Mutat 17:439-474

29. The International HapMap Consortium (2005) A haplotype map of the human genome. Nature 437:1299-1320

30. Carlson CS, Eberle MA, Rieder MJ, Yi Q, Kruglyak L, Nickerson DA (2004) Selecting a maximally informative set of single-nucleotide polymorphisms for association analyses using linkage disequilibrium. Am J Hum Genet 74:106-120

31. Schaid DJ, Rowland CM, Tines DE, Jacobson RM, Poland GA (2002) Score tests for association between traits and haplotypes when linkage phase is ambiguous. Am J Hum Genet 70:425-434

32. Lake SL, Lyon H, Tantisira K et al (2003) Estimation and tests of haplotype-environment interaction when linkage phase is ambiguous. Hum Hered 55:56-65

33. Doerge RW, Churchill GA (1996) Permutation tests for multiple loci affecting a quantitative character. Genetics 142:285-294

34. Nyholt DR (2004) A simple correction for multiple testing for single-nucleotide polymorphisms in linkage disequilibrium with each other. Am J Hum Genet 74:765-769

35. Gabriel SB, Schaffner SF, Nguyen H et al (2002) The structure of haplotype blocks in the human genome. Science 296:2225-2229

36. Barish GD, Narkar VA, Evans RM (2006) PPAR $\delta$ : a dagger in the heart of the metabolic syndrome. J Clin Invest 116:590-597

37. Shin HD, Park BL, Kim LH et al (2004) Genetic polymorphisms in peroxisome proliferator-activated receptor delta associated with obesity. Diabetes 53:847-851

38. de Bakker PIW, Burtt NP, Graham RR et al (2006) Transferability of tag SNPs in genetic association studies in multiple populations. Nat Genet 38:1298-1303

39. Montpetit A, Nelis M, Laflamme P et al (2006) An evaluation of the performance of tag SNPs derived from HapMap in a Caucasian population. PLoS Genet 2:e27 